\title{
The New (g-2) $)_{\mu}$ Experiment at Fermilab
}

\author{
Brendan C. K. Casey \\ Fermilab, PO Box 500, Batavia, IL 60510
}

\begin{abstract}
We present a proposal to measure the anomalous magnetic moment of the muon to $0.14 \mathrm{ppm}$ precision. This new g-2 experiment will be hosted by Fermilab making use of minor modifications to the existing accelerator complex. The experiment will recycle several components from the previous g-2 experiment E821 hosted at Brookhaven. In particular, the entire storage ring and magnet will be shipped to Fermilab. We cover the motivation for the experiment and review the measurement technique. We then focus on a new in-vacuo straw tracking system planned for the new experiment and its impact on searching for a permanent electric dipole moment of the muon.
\end{abstract}

Keywords: Muon, anomalous magnetic moment, electric dipole moment

PACS: 13.40.Em, 14.60.Ef

\section{INTRODUCTION}

In the last four decades, the high energy physics community has performed a series of experiments that have made precise measurements of almost every possible interaction of hadrons. Because of this, we now have a complete picture of the weak interactions among quarks at mass scales below $10 \mathrm{GeV}$. This includes fundamental properties such as lifetimes and the masses of bound states, hadron decay rates and decay properties, mixing and $\mathrm{CP}$ violation between matter and antimatter states. In the last decade, the precision at which these measurements have been made, precludes the presence of new interactions that effect flavor changing processes up to about the $\mathrm{TeV}$ scale. In many scenarios, we already have a complete picture of what flavor interactions look like beyond the electroweak symmetry breaking scale [1].

Our goal now is to have a complete picture of charged lepton interactions at the $\mathrm{TeV}$ scale at the same level of understanding as in the quark sector. This will require a large program of experiments designed to measure several types of charged lepton interactions. The $\Delta(F)=0$ interactions (where $\mathrm{F}$ indicated lepton flavor) can be probed by property measurements like $g-2$. The $\Delta(F)=1$ interactions can be probed by direct searches for lepton flavor violating processes like muon and tau decay or mu to e conversion. The $\Delta(F)=2$ processes can be studies by further limits on muonium mixing [2]. In this paper, we cover what we hope will be the first in a long series of experiments at the next generation muon sources at Fermilab and JPARC, namely the proposal to measure the muon anomalous magnetic moment to $0.14 \mathrm{ppm}$ precision [3].

\section{OVERVIEW OF THE EXPERIMENT AND REVIEW OF E821}

The experimental technique follows a long line of experiments that began at CERN, moved to Brookhaven, and are now moving to Fermilab [4]. A polarized muon beam is 
injected into a storage ring with a uniform vertical magnetic field. The V-A structure of the muon decay allows the direction of the decay positron to analyze the spin direction of the muon. Detectors are placed on the inside of the ring to count the number of positrons as a function of the time the beam has been stored. The muon spin precession around the vertical magnetic field translates into an oscillation in the positron counting rate. This oscillation frequency is proportional to the anomalous magnetic moment $a_{\mu}=(g-2) / 2$.

The E821 experiment at Brookhaven was able to understand the counting rate with to the point where the oscillation frequency could be measured for longer than 5 decades of the muon lifetime. The oscillation frequency measurement, combined with a magnetic field uniformity measured at the ppm level allowed E821 to determine the anomalous magnetic moment to $0.54 \mathrm{ppm}$ [5].

\section{Mapping out the TeV Scale with E821}

As an example of the power of these measurements, we can examine the effects the E821 result has on limiting models of new physics [3]. The measured value of $a_{\mu}$ is

larger than the expected value by $(295 \pm 81) \times 10^{-11}$. If we look at super symmetric models a guide, this deviation rules out all models that contain a negative higgs mass parameter in the Lagrangian since this would lead to a negative contribution to $a_{\mu}$. For SUSY mass scales just above the reach of LEP and the Tevatron, this would also indicate a value of $\tan \beta$ near 10 . Alternatively, the SUSY mass scale could be well above the reach of LEP and the Tevatron but this would require a very large $\tan \beta$ to account for the observed deviation to $a_{\mu}$.

It is interesting to examine this region of parameter space in greater detail. Many rare B meson processes such as $B^{+} \rightarrow \tau \nu$ and $B_{s} \rightarrow \mu^{+} \mu^{-}$receive contributions from SUSY interactions that are a function of $\tan \beta$ [6]. These decays would receive measurable contributions for values of $\tan \beta$ greater than around 50. This high $\tan \beta$-low SUSY mass region would also be seen in direct searches of super symmetric higgs bosons to tau pairs at the Tevatron [6]. Conversely, the very low regions of $\tan \beta$ have been almost completely ruled out by LEP and by tri-lepton searches at the Tevatron [7]. However, in all cases, this intermediate region of $\tan \beta$ near 10 is very hard to access in current experiments and so $g-2$ may be the best and possibly the only way to test this region independent of the LHC.

\section{G-2 AT FERMILAB}

The Tevatron program is expected to terminate some time in the next few years. At this point, the infrastructure currently used for antiproton production will be available for other purposes. The main consumer of protons at Fermilab will be the NUMI beam line which takes $150 \mathrm{GeV}$ protons from the main injector ring. The main injector ring accepts $8 \mathrm{GeV}$ protons from the booster ring and accelerates them to $150 \mathrm{GeV}$. During the acceleration, protons are still being stored at $8 \mathrm{GeV}$ in the booster that can not be used by the NUMI program. Neutrinos produced by these $8 \mathrm{GeV}$ protons will feed a low energy neutrino program consisting of miniBoone followed by microBoone. Muons 
from these $8 \mathrm{GeV}$ protons will feed a muon program beginning with g-2 and followed by the mu2e conversion experiment.

In this configuration, we expect to increase the statistics by a factor of 21 over the previous g-2 experiment at Brookhaven in approximately 2 years of shared running accumulating a total of $4 \times 10^{20}$ protons an target specifically for g-2. The current rings are close to ideal for $\mathrm{g}-2$ running and will allow for a factor of 20 reduction in the initial beam flash upon injection into the storage ring as well as a factor of 10 increase in the number of stored muons per proton on target. The repetition rate of the booster is also insures that the number of muons per fill is similar to the rate at Brookhaven.

\section{Muon EDM and In-vacuo Straw Tracking System}

As has been discussed earlier, the magnetic moment of the muon is a sensitive probe for new, TeV scale, interactions. If the new interactions also contain $\mathrm{CP}$ violating phases, these phases will give rise to an electric dipole moment (EDM) of the muon. Contrary to the muon magnetic moment, there is no EDM expected in the Standard Model at a measurable level and thus any signal indicates new sources of $\mathrm{CP}$ violation beyond the Standard Model [2]. As noted in several places, the search for extra sources of CP violation is strongly motivated since the $\mathrm{CP}$ violation observed in the quark sector so far is insufficient to explain the matter dominance of the universe [8].

If the $\mathrm{CP}$ violating phases of the new interactions are independent of lepton flavor, the muon EDM will be related to the electron EDM by simple mass scaling. In this case, the current electron EDM limits rule out the ability to discover a muon EDM with the g2 experiment proposed here. However, as discussed below, a muon EDM measurement performed with the proposed experiment could potentially extend the exclusion of flavor dependent $\mathrm{CP}$ violating interactions by 2 orders of magnitude. The phenomenology of a muon EDM is described in detail in the thesis of M. Sossong from the University of Illinois [9].

The E821 collaboration has recently submitted for publication a measurement of the muon EDM by including an up-down asymmetry, offset by $90^{\circ}$ in the $\omega_{a}$ fit yielding an upper limit of $\left|d_{\mu}\right|<1.8 \times 10^{-19} \mathrm{e} \mathrm{cm}$ [10] which is a factor of 5 improvement over the previous best limit [11]. The measurement was performed in part using straw tube tracking detectors [12] that were designed to determine the muon beam distribution within the storage ring and instrumented in front of one of the 24 calorimeter stations.

\section{Improved Traceback System}

A replication of the E821 tracking system is required for the proposed g-2 measurement. The available statistics for a muon EDM measurement could be greatly increased by instrumenting several more calorimeter stations with tracking capability. This can be accomplished by placing the straw tracking chambers inside the vacuum chambers in the scallop region in front of the calorimeters. 
A similar number of channels to the E821 traceback system can be mounted on extra vacuum ports that exist and are not instrumented in most scallop regions. A final layer can be placed outside the vacuum, directly in front of the calorimeters. While 23 additional calorimeter stations exist, several of them do not have a clear line of sight to the muon beam due to quadrapole magnets or other instrumentation so that instrumenting all stations with tracking capabilities may not be feasible.

Besides increasing the statistics for an EDM measurement, increased tracking instrumentation will allow the beam profile to be measured at several locations around the ring. This will be very useful input to beam simulation and it is expected to lead to a reduction in the error associated with the pitch correction from $0.05 \mathrm{ppm}$ to $0.03 \mathrm{ppm}$. R\&D for this straw system is ongoing at Fermilab.

\section{ACKNOWLEDGMENTS}

I would like to thank the Precision Frontier conveners, Dave Hertzog and Jim Miller for inviting me to give this present ion and the conference organizers for putting on such an excellent and productive meeting.

\section{REFERENCES}

1. M. Antonelli et al., arXiv:0907.5386v1, submitted to Phys. Rpts.

2. M. Raidal et al.,Eur. Phys. J. C57, 13 (2008).

3. R.M. Carey et al., (New g-2 Collaboration) FERMILAB-PROPOSAL-0989 (2009).

4. B.L. Roberts, arXiv:hep-ex/0510056v1.

5. G.W. Bennett et al., Phys. Rev. Lett. 92161802 (2004).

6. M. Carena, A. Melon, C.E.M. Wagner, Phys. Rev. D76 035004 (2007).

7. V. Abazov et al. (D0 Collaboration) arXiz:0901.0646v1, submitted to Phys. Lett. B

8. P. Huet, E. Sather, Phys. Rev. D51 379 (1995).

9. M.J. Sossong, A Search for an Electric Dipole Moment of the Positive Muon, Univ. of Illinois (Urbana) thesis, UMI-31-13195 (2005).

10. G.W. Bennett et al. (Muon (g-2) Collaboration), arXiv:0811.1207v1, submitted to Phys. Rev. D.

11. J. Bailey et al., j. Phys. G4, 345 (1978).

12. M. Sossong et al., submitted to Nucl. Int. Meth. A

13. C. Kendziora et al., Fermilab-Pub-02-241-E, submitted to Nucl. Int. Meth. A 